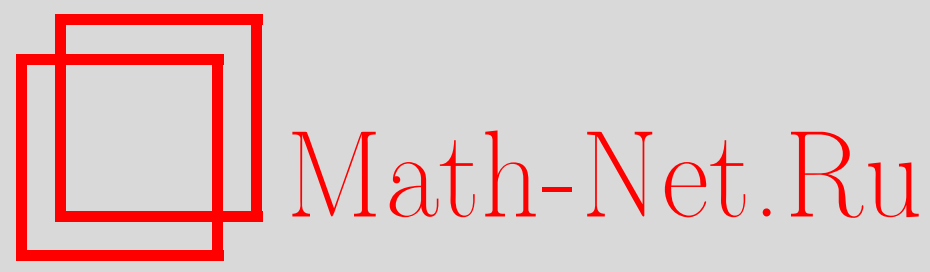

И. А. Сагиров, Степени неприводимых характеров 2-групп Судзуки, Матем. заметки, 1999, том 66, выпуск 2, 258-263

DOI: https://doi.org/10.4213/mzm1163

Использование Общероссийского математического портала Math-Net.Ru подразумевает, что вы прочитали и согласны с пользовательским соглашением http://www . mathnet.ru/rus/agreement

Параметры загрузки:

IP: 54.205 .225 .156

26 апреля 2023 г., 15:07:48

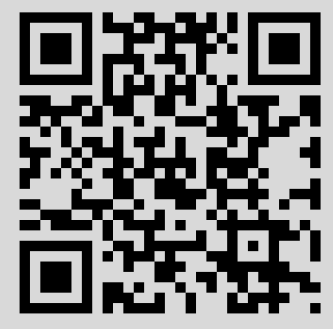


том 66 выпуск 2 Август 1999

УДК 512.54

\title{
СТЕПЕНИ НЕПРИВОДИМЫХ ХАРАКТЕРОВ 2-ГРУПП СУДЗУКИ
}

\author{
И.А. Сагиров
}

\begin{abstract}
Получено описание степеней неприводимых характеров 2-групп Судзуки $A(m, \theta)$. Пусть порядок автоморфизма $\theta$ поля $G F\left(2^{m}\right)$ равен $k>1, G=A(m, \theta), \operatorname{cd}(G)$ - множество степеней неприводимых характеров группы $G$. Если $k$ нечетно, то $\operatorname{cd}(G)=$ $\left\{1,2^{(m-m / k) / 2}\right\}$; если $k=2$, то $\operatorname{cd}(G)=\left\{1,2^{m / 2}\right\} ;$ если $k$ четно и $k \neq 2$, то $\operatorname{cd}(G)=\left\{1,2^{m / 2}, 2^{m / 2-m / k}\right\}$, причем $G$ имеет $\left(2^{m}-1\right) 2^{m / k} /\left(2^{m / k}+1\right)$ характеров степени $2^{m / 2}$ и $\left(2^{m}-1\right) 2^{2 m / k} /\left(2^{m / k}+1\right)$ характеров степени $2^{m / 2-m / k}$.

Библиографоял: 5 названий.
\end{abstract}

1. Введение. Пусть $\operatorname{Irr}(G)$ - множество всех неприводимых характеров, $\operatorname{Irr}_{1}(G)$ множество всех нелинейных неприводимых характеров, $\operatorname{cd}(G)$ - множество степеней неприводимых характеров группы $G ; \mathrm{Cl}(G)$ - множество классов сопряженньх элементов группы $G, k(G)=|\mathrm{Cl}(G)|$.

Впервые 2-групш Судзуки введены в рассмотрение Хигманом, а наиболее полно исследованы в монографии Хупшерта и Блэкберна [1, гл. VIII]. Напомним, как они определяются. Рассмотрим конечное поле $G F(q), q=2^{m}, m>1$, и его автоморфизм $\theta$ порядка $k$ для некоторого делителя $k>1$ числа $m, m / k=n$. 2-групnа Cyдзукu $A(m, \theta)$ это множество упорядоченных пар элементов из $G F(q)$ с операцией $(a, b)(c, d)=(a+c$, $b+d+c \theta(a))$ для любых $a, b, c, d \in G F(q)$. В работе получено описание степеней неприводимых характеров групп $A(m, \theta)$, а именно, доказана

Теорема. Если $G=A(m, \theta)$ - 2-группа Судзуки порядка $q^{2}$, где $m>1$ иелое, $q=2^{m}, \theta$ - автоморфизм поля $G F(q)$ порядка $k>1(m / k=n)$, то выполнено одно из следующих утверждений:

1) если $k$ нечетно, то $\operatorname{cd}(G)=\left\{1,2^{(m-n) / 2}\right\}$;

2) если $k=2, \operatorname{mo} \operatorname{cd}(G)=\left\{1,2^{m / 2}\right\}$;

3) если $k$ четно $и k \neq 2, \operatorname{mo~} \operatorname{cd}(G)=\left\{1,2^{m / 2}, 2^{m / 2-n}\right\}$, причем $G$ имеет $\left(2^{m}-1\right) 2^{n} /\left(2^{n}+1\right)$ характеров степени $2^{m / 2} u\left(2^{m}-1\right) 2^{2 n} /\left(2^{n}+1\right)$ характеров степени $2^{m / 2-n}$.

Если автоморфизм $\theta$ нечетного порядка, то соответствующий результат известен [2]. Однако, для полноты изложения мы приведем доказательство этого факта.

2. Вспомогательные результаты. Всюду далее считаем, что $G$ есть 2-групша Судзуки $A(m, \theta), \theta$ - автоморфизм порядка $k$ поля $G F(q), q=2^{m}$ и $m / k=n$. 
ЛЕмма 2.1. $\left|G^{\prime}\right| \geqslant 2^{m-n}$.

ДоКАЗАТЕЛЬСТво. Коммутатор $[(a, b),(c, d)]=(0, a \theta(c)+c \theta(a))$ для произвольных $a, b, c, d \in G F(q)$. При $c=1[(a, b),(1, d)]=(0, a+\theta(a))$ и число различных коммутаторов такого вида равно $2^{m} / u$, где $u=|\{a \in G F(q) \mid a=\theta(a)\}|$. Тогда $u$ - число неподвижных точек автоморфизма $\theta$ и $u=2^{n}$. Поэтому $\left|G^{\prime}\right| \geqslant 2^{m-n}$.

ЛЕмма 2.2. Число классов сопряженности группы $G$ равно $2^{m+n}+2^{m}-2^{n}$.

ДоказАтельство. Очевидно, $Z(G)=\{(0, b) \mid b \in G F(q)\}$ и $|Z(G)|=2^{m}$. Для произвольного элемента $(a, b) \in G, a \neq 0$, элемент $(c, d) \in C_{G}(a, b)$ тогда и только тогда, когда $[(a, b),(c, d)]=1$, т.е. $a \theta(c)+c \theta(a)=0$. Последнее означает, что $c a^{-1}=\theta\left(c a^{-1}\right)$. Так как множество неподвижных точек $\theta$ изоморфно $G F\left(2^{n}\right)$, то $\left|C_{G}(a, b)\right|=2^{m+n}$ и, следовательно, число сопряженных с произвольньм элементом из $G \backslash Z(G)$ равно $2^{m-n}$. Пусть $r$ - число нецентральных $G$-классов. Тогда $r 2^{m-n}+2^{m}=2^{2 m}=|G|$. Отсюда $r=2^{m+n}-2^{n}$ и $k(G)=2^{m+n}+2^{m}-2^{n}$.

Лемма 2.3. Отображсение $\varphi_{\lambda}(a, b)=(\lambda a, \lambda \theta(\lambda) b)$, əде $\lambda \in G F(q)^{*}$ является автоморфизмом группы $G$.

ДоказАТЕЛЬСтво. Обозначим $\lambda \theta(\lambda)=\mu$. Тогда

$$
\begin{gathered}
\varphi_{\lambda}(a, b)=(\lambda a, \mu b), \quad \varphi_{\lambda}(c, d)=(\lambda c, \mu d), \\
\varphi_{\lambda}((a, b)(c, d))=\varphi_{\lambda}(a+c, b+d+c \theta(a))=(\lambda(a+c), \mu(b+d+c \theta(a))) .
\end{gathered}
$$

С другой стороны,

$$
\begin{aligned}
\varphi_{\lambda}(a, b) \varphi_{\lambda}(c, d) & =(\lambda a, \mu b)(\lambda c, \mu d)=(\lambda a+\lambda c, \mu b+\mu d+\lambda c \theta(\lambda a)) \\
& =(\lambda(a+c), \mu b+\mu d+\mu c \theta(a))=\varphi_{\lambda}((a, b)(c, d)) .
\end{aligned}
$$

Поэтому $\varphi_{\lambda}$ - эндоморфизм групшы $G$ и $\varphi_{\lambda}(a, b)=(0,0)$ только в случае $(a, b)=(0,0)$, значит $\varphi_{\lambda}-$ автоморфизм.

Так как $\theta$ - автоморфизм поля $G F(q)$ порядка $k$, для любого $x \in G F(q) \quad \theta(x)=x^{2^{n t}}$ с некоторым $t,(t, k)=1$. Зафиксируем это $t$.

ЛЕмма 2.4. Если $m=n k-$ произведение двух натуральных чисел и число $t$ таково, что $(t, k)=1$, то

$$
\left(2^{m}-1,2^{n t}+1\right)= \begin{cases}1, & \text { если k нечетно } \\ 2^{n}+1, & \text { если k четно }\end{cases}
$$

ДокАЗАТЕльСтво. Как известно, $\left(p^{m}-1, p^{n}-1\right)=p^{(m, n)}-1$ для любых $m, n \in \mathbb{N}$ и простого $p$. Поэтому

$$
\left(2^{m}-1,2^{2 n t}-1\right)= \begin{cases}2^{n}-1, & \text { если } k \text { нечетно, } \\ 2^{2 n}-1, & \text { если } k \text { четно. }\end{cases}
$$

Очевидно, $\left(2^{m}-1,2^{n t}+1\right) \mid\left(2^{m}-1,2^{2 n t}-1\right)$.

Заметим, что $\left(2^{n t}+1,2^{n}-1\right)=1$. Действительно, если $p \mid\left(2^{n t}+1,2^{n}-1\right)$ и $p$ простое, то $2^{n} \equiv 1(\bmod p)$, а значит, $2^{n t} \equiv 1^{t}(\bmod p)$. Получили противоречие, так как $2^{n t} \equiv-1(\bmod p)$. Поэтому при нечетном $k\left(2^{m}-1,2^{n t}+1\right)=1$. В силу того что $2^{2 n}-1=\left(2^{n}-1\right)\left(2^{n}+1\right),\left(2^{n t}+1,2^{n}-1\right)=1$ и $2^{n}+1 \mid 2^{n t}+1$, при четном $k$ $\left(2^{m}-1,2^{n t}+1\right)=2^{n}+1$. 
ЛЕмма 2.5. Пусть $\lambda$ - примитивный әлемент поля $G F(q)$. Длина орбиты $\varphi_{\lambda}$ на $G \backslash Z(G)$ равна $2^{m}-1$. Длина орбиты $\varphi_{\lambda}$ на $Z(G)^{\#}$ равна $2^{m}-1$ при нечетном $k$ $u\left(2^{m}-1\right) /\left(2^{n}+1\right)$ при четном $k$.

ДокаЗАтЕЛьСтво. Для любого $(a, b) \in G \varphi_{\lambda}^{i}(a, b)=\left(\lambda^{i},(\lambda \theta(\lambda))^{i} b\right)$. Пусть $a \neq 0$. Тогда $\varphi_{\lambda}^{i}(a, b)=(a, b)$ в том и только том случае, когда $\left(\lambda^{i}-1\right) a=0$, что невозможно при $0<i<2^{m}-1$.

Пусть $a=0$. Тогда $\varphi_{\lambda}^{i}(0, b)=\left(0,(\lambda \theta(\lambda))^{i} b\right)=(0, b)$ только при $(\lambda \theta(\lambda))^{i}=1$. Так как $\theta(\lambda)=\lambda^{2^{n t}}$, последнее вьполнено при $\lambda^{\left(2^{n t}+1\right) i}=1$. В силу леммы 2.4 при нечетном $k$ отсюда следует, что $2^{m}-1 \mid i$ и все элементы $Z(G)^{\#}$ сопряжены под действием $\varphi_{\lambda}$. При четном $k\left(2^{m}-1\right) /\left(2^{n}+1\right) \mid i$ и длина $\varphi_{\lambda}$-орбиты элемента $(0, b)$ равна $\left(2^{m}-1\right) /\left(2^{n}+1\right)$.

СлЕДСТВИЕ. При нечетном $k>1 G^{\prime}=Z(G)$.

ЛЕмма 2.6 [3], [4]. Если порядок коммутанта 2-группы $G$ с ииклической подгруппой Фраттини равен 2, то $G=(B * C) \times E_{2^{s}}$, где $B$ әкстраспециальна или единична, $C$ полуабелева или ииклична, причем если $B$ единична, то $C$ полуабелева; $s \geqslant 0$.

ЗАмЕЧАНИЕ. Если в лемме $2.6|\Phi(G)|=2$, то, в силу того что порядок подгруппы $\Phi$ раттини полуабелевой 2-групшы больше $2, C$ - циклическая группа.

Лемма 2.7. Пусть $\chi \in \operatorname{Irr}_{1}(G), \chi(1)=2^{a}, Z_{0}=Z(G) \cap \operatorname{Ker} \chi$. Тогдa $\left|Z(G): Z_{0}\right|=2$, $G^{\prime} \nless Z_{0}, \operatorname{cd}\left(G / Z_{0}\right)=\left\{1,2^{a}\right\},\left|\operatorname{Irr}_{1}\left(G / Z_{0}\right)\right|=2^{m-2 a}$. Кроме того, любой нелинейный неприводимый характер $G$ является характером ровно одной группь $G / Z$, где $|Z(G): Z|=2 u G^{\prime} \nless Z$.

ДоказАтЕльство. Так как $Z(G / \operatorname{Ker} \chi)$ - циклическая группа, а $Z(G)$ - элементарная абелева группа, то $\left|Z(G): Z_{0}\right|=2$. Рассмотрим факторгрупшу $G / Z_{0}$. Так как $\left|\left(G / Z_{0}\right)^{\prime}\right|=\left|\Phi\left(G / Z_{0}\right)\right|=2$, то в силу леммы 2.6 и замечания $G / Z_{0}=(B * C) \times E_{2^{s}}$, где $B$ экстраспециальная, а $C$ циклическая. В силу того что $\chi \in \operatorname{Irr}_{1}\left(G / Z_{0}\right)$, вьполнено $|B|=$ $2^{2 a+1}$ и $\operatorname{cd}\left(G / Z_{0}\right)=\left\{1,2^{a}\right\}$. Так как $\left|G / Z_{0}\right|=2^{m+1}$, то $\left|\operatorname{Irr}_{1}\left(G / Z_{0}\right)\right|=2^{m-2 a}$. Последнее утверждение леммы очевидно, так как если $\chi$ является характером групп $G / Z_{1}$ и $G / Z_{2}\left(\left|Z(G): Z_{i}\right|=2\right)$ одновременно, то $Z(G) \leqslant \operatorname{Ker} \chi$, а значит, $G^{\prime} \leqslant \operatorname{Ker} \chi$ и $\chi$ линеен.

\section{3. Доказательство основных результатов.}

Теорема 3.1. Если $G=A(m, \theta)$ - 2-группа Судзуки и $\theta$ - автоморфизм поля $G F(q)$ нечетного порядка $k>1, \operatorname{mo} \operatorname{cd}(G)=\left\{1,2^{(m-n) / 2}\right\}$.

ДоКАЗАТЕЛЬСтво. Пусть $\lambda$-примитивный элемент поля $G F(q)$. Рассмотрим действие автоморфизма $\varphi_{\lambda}$ на подгруппах индекса 2 в $Z(G)$. Предположим, что существуют подгрупша $Z_{0}$ индекса 2 в $Z(G)$ и число $l, 0<l<2^{m}-1$, такие, что $Z_{0} \varphi_{\lambda}^{l}$-допустима. Рассмотрим $Z(G)$ как векторное пространство размерности $m$ над $G F(2)$. Тогда $\varphi_{\lambda}^{l}-$ автоморфизм этого пространства, $Z_{0}-\varphi_{\lambda}^{l}$-допустимое подпространство и $\varphi_{\lambda}^{l}$ нечетного порядка. Тогда по теореме Машке существует элемент $(0, z) \in Z(G)$ \# такой, что $Z(G)=Z_{0} \times\langle(0, z)\rangle$ и $\varphi_{\lambda}^{l}(0, z)=(0, z)$. Это противоречит лемме 2.5 , так как при нечетном $k$ длина орбиты $(0, z)$ под действием $\varphi_{\lambda}$ равна $2^{m}-1$, а значит, $\varphi_{\lambda}^{l}$ не имеет неподвижных точек. 
Следовательно, $\left\{\varphi_{\lambda}^{i}\left(Z_{0}\right)\left|0<i \leqslant 2^{m}-1,\right| Z(G): Z_{0} \mid=2\right\}$ - множество всех подгрупп индекса 2 в $Z(G)$. Так как каждьй нелинейньй неприводимьй характер группы $G$ принадлежит ровно одной группе $G / \varphi_{\lambda}^{i}\left(Z_{0}\right)$ (лемма 2.7 ), а они все изоморфны, то $|\operatorname{cd}(G)|=2$.

Пусть $\operatorname{cd}(G)=\{1, d\}$. Так как $|\operatorname{Lin}(G)|=2^{m}$ (следствие из леммы 2.5), а $G$ имеет $2^{m+n}-2^{n}$ нелинейных характеров, то $2^{m}+\left(2^{m+n}-2^{n}\right) d^{2}=2^{2 m}$ и $d=2^{(m-n) / 2}$.

В дальнейшем будем считать, что $k$ четно.

Лемма 3.2. Пусть $\lambda$ - примитивный әлемент поля $G F(q)$. Тогда

1) если $m \neq 2 n$, то $G^{\prime}=Z(G)$ u $\left(G^{\prime}\right)^{\#}$ имеет $2^{n}+1 \varphi_{\lambda}$-орбит длинь $\left(2^{m}-1\right) /\left(2^{n}+1\right)$

2) если $m=2 n$, то либо $G^{\prime}=Z(G)$ и $\left(G^{\prime}\right)^{\#}$ имеет $2^{n}+1 \quad \varphi_{\lambda}$-орбит длинь $\left(2^{m}-1\right) /\left(2^{n}+1\right)=2^{n}-1$, либо $\left|G^{\prime}\right|=2^{m / 2}$ и все әлементы $\left(G^{\prime}\right)^{\#}$ сопря⿻сены под действием автоморфизма $\varphi_{\lambda}$.

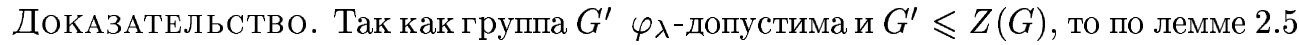
элементы $\left(G^{\prime}\right)^{\#}$ разбиваются под действием $\varphi_{\lambda}$ на орбиты длины $\left(2^{m}-1\right) /\left(2^{n}+1\right)$. Пусть число $\varphi_{\lambda}$-орбит элементов $\left(G^{\prime}\right)^{\#}$ равно $l$, а $\left|G^{\prime}\right|=2^{f}$. Тогда $2^{f}=l\left(2^{m}-1\right) /$ $\left(2^{n}+1\right)+1$, откуда $l-1=l 2^{m}+2^{n}-2^{f}-2^{f+n}$. Так как $\left|G^{\prime}\right| \geqslant 2^{m-n}$ (лемма 2.1), то $2^{n} \mid 2^{f}$ и $2^{n} \mid l-1$.

Поскольку $\left|G^{\prime}\right| \leqslant 2^{m}$, либо $l=2^{n}+1$ и $\left|G^{\prime}\right|=2^{m}$, либо $l=1$ и $\left|G^{\prime}\right|=2^{f}=\left(2^{m}-1\right) /$ $\left(2^{n}+1\right)+1$. Вовтором случае $2^{f}\left(2^{n}+1\right)=2^{n}\left(2^{m-n}+1\right)$, а значит, $m=2 n$ и $\left|G^{\prime}\right|=2^{m / 2}$.

Будем считать, что $G^{\prime}=Z(G)$, т.е. элементы $\left(G^{\prime}\right)^{\#}$ разбиваются под действием $\varphi_{\lambda}$ на $2^{n}+1$ орбит длины $\left(2^{m}-1\right) /\left(2^{n}+1\right)$.

Лемма 3.3. Пусть $\lambda$ - примитивный әлемент поля $G F(q)$. Тогда

1) множество подгрупп индекса 2 в $Z(G)$ имеет $2^{n}+1 \quad \varphi_{\lambda}$-орбит длинь $\left(2^{m}-1\right) /\left(2^{n}+1\right)$

2) под действием автоморфизма $\varphi_{\lambda}$ неглавные неприводимые характеры $G$ разбиваются на $2^{n}$ орбит длины $2^{m}-1$ и $2^{n}+1$ орбит длины $\left(2^{m}-1\right) /\left(2^{n}+1\right)$.

ДокАЗАТЕЛЬСтво. 1) По лемме $2.5\left|\varphi_{\lambda}\right|_{Z(G)} \mid=\left(2^{m}-1\right) /\left(2^{n}+1\right)$. Пусть $Z_{0} \leqslant Z(G)$, $\left|Z(G): Z_{0}\right|=2$. Предположим, что $Z_{0}^{\varphi_{\lambda}^{i}}=Z_{0}$ для некоторого $0<i<\left(2^{m}-1\right) /\left(2^{n}+1\right)$. Так как $\left(\left|\varphi_{\lambda}\right|,|Z(G)|\right)=1$, по теореме Машке существует элемент $(0, z) \in Z(G)$ \# такой, что $Z(G)=Z_{0} \times\langle(0, z)\rangle$ и $\varphi_{\lambda}^{i}(0, z)=(0, z)$, а это противоречит лемме 2.5. Таким образом, длины $\varphi_{\lambda}$-орбит подгрупп индекса 2 в $Z(G)$ равны $\left(2^{m}-1\right) /\left(2^{n}+1\right)$, а число орбит $-2^{n}+1$.

2) Определим действие $\varphi_{\lambda}$ на $\operatorname{Irr}(G)$. Пусть $\chi \in \operatorname{Irr}(G)$ и $R$ - представление $G$ с характером $\chi$. Тогда $R \varphi_{\lambda}^{-1}$ - также представление $G$, и его характер определяется как $\chi^{\varphi_{\lambda}}(g)=\chi\left(g^{\varphi_{\lambda}^{-1}}\right)$ для любого $g \in G$. Очевидно, $\chi^{\varphi_{\lambda}} \in \operatorname{Irr}(G)$.

Пусть $X=\left(\chi_{i}\left(K_{j}\right)\right)$ - таблица характеров $G$, где $\left\{K_{1}, K_{2}, \ldots, K_{r}\right\}=\mathrm{Cl}(G)$, $r=2^{m+n}+2^{m}-2^{n}$. Тогда $\varphi_{\lambda}$ действует на столбцах $X$ подстановкой $\pi_{1}$, а на строках подстановкой $\pi_{2}$. Причем если рассматривать $\pi_{1}$ и $\pi_{2}$ как элементы GL $(r, \mathbb{C})$, то $\pi_{2} X=\left(\chi_{i}^{\varphi_{\lambda}}\left(K_{j}\right)\right)=\left(\chi_{i}\left(K_{j}^{\varphi_{\lambda}^{-1}}\right)\right)=X \pi_{1}$. Так как $X-$ невырожденная матрица, то $\pi_{1}=X^{-1} \pi_{2} X$ в $\mathrm{GL}(r, \mathbb{C})$. Тогда согласно лемме Вишневецкого $[5$, гл. $\mathrm{X}] \pi_{1}$ и $\pi_{2}$ сопряжены в $S_{r}$, а значит, имеют одинаковое цикленное строение. Действие $\varphi_{\lambda}$ на 
классах сопряженности из $G^{\#}$ известно: $2^{n}+1$ орбит длины $\left(2^{m}-1\right) /\left(2^{n}+1\right)$ на центральных классах и $2^{n}$ орбит длины $2^{m}-1$ на нецентральных.

Лемма 3.4. Пусть $\chi \in \operatorname{Irr}_{1}(G), \chi(1)=2^{a}$. Тогда $m / 2-n \leqslant a \leqslant m / 2$.

ДокАЗАтЕЛьСтво. По лемме $2.7 \chi \in \operatorname{Irr}_{1}\left(G / Z_{0}\right)$, где $Z_{0} \leqslant Z(G),\left|Z(G): Z_{0}\right|=2$ и $\left|\operatorname{Irr}_{1}\left(G / Z_{0}\right)\right|=2^{m-2 a}$. Последнее означает, что $m-2 a \geqslant 0$ и $a \leqslant m / 2$.

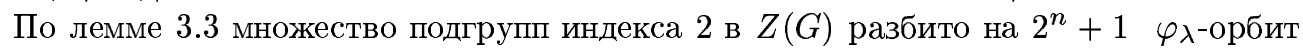
длины $\left(2^{m}-1\right) /\left(2^{n}+1\right)$. Пусть $Z_{1}, \ldots, Z_{2^{n}+1}$ - представители орбит. Тогда по лемме $2.7 \operatorname{cd}\left(G / Z_{i}\right)=\left\{1,2^{a_{i}}\right\},\left|\operatorname{Irr}_{1}\left(G / Z_{i}\right)\right|=2^{m-2 a_{i}}$. Кроме того, любые две подгруппы индекса 2 в $Z(G)$, принадлежащие одной $\varphi_{\lambda}$-орбите, изоморфны. Так как $\left|\operatorname{Irr}_{1}(G)\right|=2^{m+n}-2^{n}$, то

$$
2^{m+n}-2^{n}=\frac{2^{m}-1}{2^{n}+1} \sum_{i=1}^{2^{n}+1} 2^{m-2 a_{i}}
$$

откуда

$$
\sum_{i=1}^{2^{n}+1} 2^{m-2 a_{i}}=2^{n}\left(2^{n}+1\right)
$$

Предположим, что $m-2 a_{i}>2 n$ для некоторого $i$. Тогда $2^{m-2 a_{i}} \geqslant 2^{2 n+1}=2^{2 n}+2^{2 n}>$ $2^{n}\left(2^{n}+1\right)$. Получили противоречие. Следовательно, $m-2 a_{i} \leqslant 2 n$ и $a_{i} \geqslant m / 2-n$ для любого $i$.

Tеорема 3.5. Если $G^{\prime}=Z(G), \operatorname{mo} \operatorname{cd}(G)=\left\{1,2^{m / 2-n}, 2^{m / 2}\right\}$. Причем группа $G$ имеет $\left(2^{m}-1\right) 2^{2 n} /\left(2^{n}+1\right)$ неприводимых характеров степени $2^{m / 2-n} u$ $\left(2^{m}-1\right) 2^{n} /\left(2^{n}+1\right)$ неприводимых характеров степени $2^{m / 2}$.

ДокАЗАТЕЛЬСТВо. Обозначим $\left(2^{m}-1\right) /\left(2^{n}+1\right)=s$.

По лемме 3.3 неглавные неприводимые характеры $G$ под действием $\varphi_{\lambda}$ разбиваются на $2^{n}$ орбит длины $2^{m}-1$ и $2^{n}+1$ орбит длины $s$. Заметим, что в силу $\operatorname{Lin}(G) \cong G / G^{\prime}$ неглавные линейные характеры $G$ образуют одну $\varphi_{\lambda}$-орбиту длины $2^{m}-1$. Поэтому на $\operatorname{Irr}_{1}(G)$ будет $2^{n}-1 \varphi_{\lambda}$-орбит длины $2^{m}-1$. По лемме 2.7 каждый нелинейный неприводимый характер $G$ является характером ровно одной групшы $G / Z_{0}$, где $Z_{0} \leqslant Z(G)$ и $\left|Z(G): Z_{0}\right|=2$.

По лемме $3.3 \varphi_{\lambda}$ имеет $2^{n}+1$ орбит длины $s$ на множестве подгрупп индекса 2 в $Z(G)$. Тогда для любой подгруппы $Z_{0}$ индекса 2 в $Z(G)$ выполняется $Z_{0}^{\varphi_{\lambda}^{s}}=Z_{0}$. Пусть длина $\varphi_{\lambda}$-орбиты характера $\chi \in \operatorname{Irr}_{1}(G)$ равна $2^{m}-1$ и $\chi \in \operatorname{Irr}_{1}\left(G / Z_{0}\right)$. Тогда $\chi_{\lambda}^{\varphi_{\lambda}} \in$ $\operatorname{Irr}_{1}\left(G / Z_{0}\right), 0 \leqslant i<2^{n}+1$, но $\chi_{\lambda}^{\varphi_{\lambda}^{s i}} \neq \chi$. Это означает, что в $G / Z_{0}$ имеется $2^{n}+1$ характеров, сопряженных с $\chi$ под действием $\varphi_{\lambda}$.

Предположим, что для некоторой групшы $Z_{0} \leqslant Z(G),\left|Z(G): Z_{0}\right|=2$, в $G / Z_{0}$ нет неприводимых характеров, длина $\varphi_{\lambda}$-орбиты которых равна $s$. Но тогда нелинейные неприводимые характеры $G / Z_{0}$ разбиты на $\varphi_{\lambda}^{s}$-орбиты длины $2^{n}+1$, и если $\operatorname{cd}\left(G / Z_{0}\right)=$ $\left\{1,2^{a}\right\}$, то по лемме $2.72^{n}+1 \mid 2^{m-2 a},-$ противоречие. Значит, в каждой групе $G / Z_{0}$ существует по крайней мере один характер, длина орбиты которого равна $s$. Так как

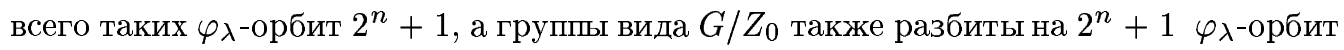
длины $s$, то в каждой подгруппе $G / Z_{0}$ ровно один характер искомого типа. 


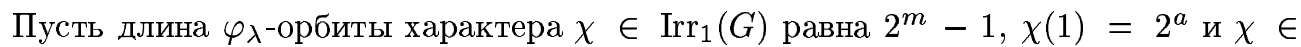
$\operatorname{Irr}_{1}\left(G / Z_{0}\right)$. Тогда по доказанному выше $2^{m-2 a}=1+l\left(2^{n}+1\right), l>0$. Отсюда следует, что $2^{n}+1 \mid 2^{m-2 a}-1$, а значит, $n \mid m-2 a$ и $(m-2 a) / n$ четно. Однако, по лемме $3.4 m-2 n \leqslant 2 a \leqslant m$ и $0 \leqslant(m-2 a) / n \leqslant 2$, т.е. $(m-2 a) / n \in\{0,2\}$. Поскольку $l>0$, равенство $(m-2 a) / n=0$ невозможно. Значит, $a=m / 2-n$ и в $G / Z_{0}$ $2^{2 n}$ нелинейных характеров, которые образуют $2^{n}-1 \varphi_{\lambda}$-орбит длины $2^{m}-1$ и одну орбиту длины $s$. Таким образом, все $\varphi_{\lambda}$-орбиты длины $2^{m}-1$ исчерпаны, и если $Z_{1} \notin\left\{Z \leqslant Z(G),|Z(G): Z|=2 \mid Z=Z_{0}^{\varphi_{\lambda}^{i}}\right\}$, то в $G / Z_{1}$ ровно один нелинейньй характер степени $2^{m / 2}$.

Итак, $\operatorname{cd}(G)=\left\{1,2^{m / 2-n}, 2^{m / 2}\right\}$. Причем группа $G$ имеет $\left(2^{m}-1\right) 2^{2 n} /\left(2^{n}+1\right)$ неприводимых характеров степени $2^{m / 2-n}$ и $\left(2^{m}-1\right) 2^{n} /\left(2^{n}+1\right)$ неприводимых характеров степени $2^{m / 2}$.

СлЕДСТВИЕ. Если $m=2 n, m o\left|G^{\prime}\right|=2^{m / 2}$.

ДокАЗАТЕльство. Действительно, в этом случае по лемме 3.2 либо $\left|G^{\prime}\right|=2^{m / 2}$, либо $\left|G^{\prime}\right|=2^{m}$. Но во втором случае по теореме $3.5 \operatorname{cd}(G)=\left\{1,2^{m / 2-n}, 2^{m / 2}\right\}$, что невозможно в силу $m / 2-n=0$.

TEOPEMA 3.6. Ecлu $m=2 n, \operatorname{mo} \operatorname{cd}(G)=\left\{1,2^{m / 2}\right\}$.

ДокАЗАТЕЛЬСтво. По следствию из теоремы $3.5\left|G^{\prime}\right|=2^{m / 2}$, а по лемме 3.2 у $\varphi_{\lambda}$ на $\left(G^{\prime}\right)$ \# одна орбита длины $2^{m / 2}-1$. По лемме 2.7 любой нелинейный неприводимьй характер $G$ принадлежит ровно одной групше $G / Z_{0}$, где $G^{\prime} \nless Z_{0} \leqslant Z(G)$ и $\left|Z(G): Z_{0}\right|=2$. Число групп такого вида $2^{m / 2}-1$, и все они сопряжены под действием $\varphi_{\lambda}$ (доказательство аналогично лемме 3.3). Поэтому все групшы типа $G / Z_{0}$ изоморфны и $|c d(G)|=2$. По лемме 2.2 число классов сопряженности группы $G$ равно $2^{m+n}+2^{m}-2^{n}$. Пусть $\operatorname{cd}(G)=\{1, d\}$. Тогда $|G|=2^{2 m}=2^{m+m / 2}+d^{2}\left(2^{m}-2^{m / 2}\right)$, откуда $d=2^{m / 2}$.

\section{СПИСОК ЦИТИРОВАННОЙ ЛИТЕРАТУРЫ}

[1] Huppert B., Blackburn N. Finite Groups II. Berlin-Heidelberg-New York: Springer, 1982.

[2] Hanaki A. A condition on lengths of conjugacy classes and character degrees // Osaka J. Math. 1996. V. 33. P. 207-216.

[3] Беркович Я. Г. Обобщение теорем $\Phi$. Холла и Н. Блэкберна и их применение к нерегулярньп $p$-группам // Изв. АН СССР. Сер. матем. 1971. Т. 35. № 4. С. 800-830.

[4] Старостин А. И. Конечные 2-группы с циклической подгруппой Фраттини // Тр. ИММ. Т. 3. Екатеринбург: УрО РАН, 1995. С. 60-64.

[5] Berkovich Ya.G., Zhmud' E. M. Characters of Finite Groups. 1. Transl. Math. Monographs. V. 172. Providence (R.I.): Amer. Math. Soc., 1997. 